\title{
I congressi internazionali IAH: da Marrakech 2014 verso Roma 2015
}

Marco Petitta - Presidente del Comitato Italiano IAH - marco.petitta@uniroma1.it Daniela Ducci - Segretario del Comitato Italiano IAH - segretario@iahitaly.it

Nel 2014 il congresso internazionale della IAH si è tenuto per la prima volta in Nord Africa, infatti dal 15 al 19 settembre a Marrakech in Marocco si sono incontrati più di 400 idrogeologi provenienti da tutto il mondo nella splendida e suggestiva cornice del Ryad Mogador Hotel.

Nella sessione di apertura, c'è stato il benvenuto di una idrogeologa di eccezione: Madame Charafat Afilal, Segretario di Stato per il Dipartimento Acqua (Ministero dell'Energia, delle Miniere, dell'Acqua e dell'Ambiente) che ha ricordato l'importanza delle acque sotterranee in Marocco e sottolineato la necessità di gestire correttamente la risorsa idrica.

La conferenza si è articolata in 10 temi principali (T1: Climate change and groundwater resources, T2: Interaction groundwater / surface water, T3: Vulnerability, pollution and rebabilitation of groundwater resources, T4: Aquifers in fractured and karstic environments, T5: Coastal aquifers, T6: Management and governance of groundwater resources, T7: Tools and techniques for the investigation of groundwater resources, T8: Unconventional groundwater resources, T9 : Hydrogeology of arid zones, T10: Groundwater and oil exploitation) ognuno associato a varie sessioni tecniche. Infatti il calendario della settimana è proseguito con 17 keynote lectures, le sessioni parallele e i poster. Tra le keynotes in sessione plenaria è emersa sicuramente quella di Richard Taylor che ha illustrato le relazioni in clima arido fra bilanci idrogeologici e cambiamenti climatici.

Le sessioni tecniche si sono articolate su quattro giorni con quasi 300 presentazioni e poco più di 100 poster, divisi nelle sessioni parallele. Il mercoledì, come prassi nei congressi IAH, è stato dedicato ai visite tecniche, con cinque diverse opzioni, focalizzate su sorgenti, miniere, dighe, etc.

Come sempre inoltre c'è stato spazio per le riunioni del Consiglio di IAH, l'Assemblea Generale Annuale, le riunioni delle Commissioni e network e la riunione dell'Early Career Hydrogeologist's Network (ECHN) che ha assegnato, come è ormai tradizione, i premi per la miglior presentazione ed il miglior poster dei giovani idrogeologi. Vari gli eventi sociali, tra cui lo spettacolare gala dinner tenutosi nel complesso turistico Chez Ali, sotto gli enormi tendoni del palmeto di Marrakech con il personale in costume tradizionale e musicisti al tavolo. Al termine della cena c'è stata l'esibizione di acrobati e ballerine di danza del ventre con corse di cavalli berberi montati da abili cavalieri. Alla fine sorprendente lo spettacolo pirotecnico, con un gran finale dedicato proprio al congresso IAH 2014.

Una nuova avventura dell'IAH internazionale (iniziativa congiunta tra la Segretaria IAH e l'ECHN) è stata presentata ed è cominciata in questo congresso: lo scambio e il dono dei libri di Idrogeologia. Sono stati visti delegati anziani arrivare presso lo stand IAH con trolley pieni di libri da mettere a disposizione degli idrogeologi più giovani e questi ultimi nella affannosa ricerca del testo più cool..... in conclusione giovedì all'ora di pranzo tutti i libri erano stati presi. Questa riuscitissima iniziativa verrà certamente replicata nei futuri congressi IAH.

Dopo il congresso sono partiti tre field trip post-congresso, che hanno avuto molto successo, sia pure con qualche inconveniente per i partecipanti (maltempo e ritardi vari).

La comunità idrogeologica italiana è stata presente con più di venti partecipanti, per la maggior parte giovani, che hanno illustrato brillantemente con interventi orali e poster i risultati delle loro ricerche. Durante il convegno inoltre alcuni membri della delegazione italiana hanno partecipato a varie sessioni in qualità di convenor e ai vari meeting, interagendo sia con i colleghi marocchini che con quelli di tutto il mondo. In particolare all'assemblea dell'IAH è stato annunciato il Congresso di Roma AQUA2015 ed è stato presentato con grande successo il cortometraggio promozionale su Roma composto da vari spezzoni di film girati nella capitale, da "La dolce vita" a "La grande bellezza", accompagnati dalle note nostalgiche di Domenico Modugno e Adriano Celentano.

Quale è stata alla fine l'impressione globale sul Congresso? Certamente i piacevoli eventi sociali hanno favorito gli scambi scientifici tra i delegati e la qualità scientifica, sia pure di livello inferiore rispetto agli anni precedenti, ha fatto registrare alcuni spunti di notevole interesse. Sicuramente la maggior parte dei partecipanti avrà lasciato Marrakech con un ottimo ricordo del Congresso ma ...con tanti ostacoli. Infatti la piena riuscita del Congresso è stata pregiudicata dalla disorganizzazione, sia nella logistica (vedi soprattutto problemi alberghieri, location remota degli stands, field trip, etc.), che nelle sessioni scientifiche: talora le assenze di oratori e anche di convenors hanno creato momenti imbarazzanti ed è stato difficile se non impossibile riuscire a farsi una programmazione personale e trovare le varie sedi delle sessioni (scarse o nulle le indicazioni).

Questi errori, del tutto giustificabili per la difficoltà di organizzare una conferenza internazionale di tali dimensioni per un piccolo Chapter con scarse risorse, devono essere un monito e un insegnamento per l'organizzazione di AQUA2015, ormai alle porte.

Facendo tesoro dell'esperienza marocchina e guardando agli ultimi successi dei congressi di Niagara Falls e Perth, il Comitato Organizzatore sta lavorando ormai a pieno ritmo per assicurare la buona riuscita del prossimo congresso, previsto a Roma dal 13 al 18 settembre 2015 .

La sottomissione degli abstracts è aperta fino al 31 marzo, tramite la piattaforma on-line del sito www.iah2015.org. Prima di Natale è stata aperta anche la pagina per la registrazione, che prevede fino al 15 giugno il pagamento di una quota ridotta sia per i soci IAH che per i non soci. E' quindi 
già possibile iscriversi e prenotare anche gli alberghi tramite la procedura di iscrizione on-line. Sono anche state pubblicate le istruzioni per la realizzazione dei poster elettronici, una novità assoluta per i congressi IAH. Al posto dei poster tradizionali, verranno utilizzati degli schermi elettronici dove i poster in formato PDF verranno preventivamente caricati, con la possibilità di includere filmati relativi a esperimenti di laboratorio o di campo, simulazioni grafiche e quant'altro possa aiutare ad esporre il proprio lavoro.

Analogo sforzo si è concentrato sul programma degli eventi sociali, che si aprirà con il classico welcome cocktail nel pomeriggio di domenica 13 settembre presso l'Angelicum, sede del congresso per tutta la settimana. Il lunedì sera sarà la volta dell'evento organizzato a cura dell'ECHN, con invito esteso non solo ai giovani idrogeologi ma a tutti i partecipanti. La sera successiva ci sarà l'opportunità di partecipare ad una cena sociale nel centro di Roma, in prossimità del Pantheon, presso il ristorante Shari Vari, da unire ad una passeggiata serale nel cuore della città eterna. L'evento principale del congresso, incluso nella quota di iscrizione, si terrà il giovedì sera nella cornice di EATALY, presso la Stazione Ostiense, dove è stato riservato il terzo piano del complesso per una serata all'insegna del cibo italiano di qualità. A completare gli eventi sociali, il programma previsto per gli accompagnatori, che oltre al welcome cocktail e alla serata da EATALY, include due tour turistici organizzati nella Roma imperiale e alla Cappella Sistina e Musei Vaticani, e il mid week field trip.

A tale proposito, nel solco della tradizione IAH, il mercoledì sarà totalmente dedicato alle escursioni tecniche, che prevedono l'offerta di ben otto alternative: dal tour dentro Roma alla scoperta degli acquedotti alle acque termali del viterbese, dal delta del Tevere all'Appennino reatino, dalla Pianura Pontina ai bagni di Tivoli, dalla piana del Fucino alle sorgenti di Riardo. Con l'aggiunta, per chi non volesse muoversi da Roma, dell'opportunità di partecipare all'udienza di Papa Francesco in Vaticano. Il venerdì sera, dopo la chiusura del congresso, partiranno i tour post-congresso, di durata compresa tra i quattro e i sei giorni, che faranno conoscere oltre alle bellezze paesaggistiche e artistiche anche l'idrogeologia italiana: le mete saranno Napoli e Ischia, la Toscana, l'Appennino Abruzzese-Campano, le Alpi Occidentali, la laguna Veneta e il delta del Po o infine la Puglia.

Il cuore del congresso sarà ovviamente il programma tecnico, che si aprirà con i corsi pre-congresso, che occuperanno da uno a tre giorni prima dell'inizio dei lavori: saranno offerti corsi sull'idrogeologia dei contaminanti, sulla modellistica idrogeologica, sull'uso dei GIS e su altri argomenti tecnici di grande richiamo. Il programma scientifico, recentemente pubblicato sul sito, prevede otto argomenti principali (Topic), ognuno suddiviso in un numero variabile di sessioni, per un totale di 68 potenziali sessioni, che verranno attivate in funzione del numero di riassunti che perverranno da qui a fine marzo. Per il primo tema (Groundwater, food and health), la collaborazione con UNESCO e FAO consentirà di aprire il congresso a problematiche non sempre affrontate in precedenza. Più classico il secondo tema (Groundwater flow systems beha- viour), che include sessioni che spaziano dal mondo carsico alle sorgenti termali e minerali, dal bilancio idrogeologico fino alle crisi idriche. Il terzo tema sarà dedicato all'uso sostenibile della risorsa idrica (Sustainable use of groundwater resources), con focus tra l'altro sui cambiamenti climatici, sulla protezione degli acquiferi, sulla ricarica artificiale e sul potenziale geotermico. Il quarto tema sarà dedicato agli impatti antropici (Urban and contaminant bydrogeology), dalla caratterizzazione fino alla bonifica delle falde, passando per i contaminanti emergenti e per i reflui minerari. La particolare attenzione alle problematiche ambientali è contenuta nel quinto tema (Ground/Surface water: an integrated view), contenente sessioni, tra le altre, sugli ecosistemi dipendenti dalle acque sotterranee, sulla gestione integrata della risorsa idrica, sugli impatti delle variazioni climatiche. Il governo delle acque sotterranee è il filo conduttore del sesto tema (Groundwater governance and Policy), dagli acquiferi transfrontalieri alla implementazione delle normative, dalla vulnerabilità degli acquiferi agli aspetti legali ed economici della risorsa idrica. Il settimo tema è concentrato sulle aree costiere (Groundwater management in coastal areas), comprendendo non solo l'intrusione marina, ma i problemi della pressione antropica e quello degli impatti ecologici, fino alla modellistica densità-dipendente. L'ottavo e ultimo tema sarà rivolto al futuro dell'idrogeologia (New tools and new frontiers), con una varietà di sessioni dai modelli all'uso degli isotopi, dall'idrogeofisica alle problematiche energetiche, dal fracking fino alle nanotecnologie e via discorrendo. In definitiva, un programma talmente ricco da diventare imperdibile per chiunque voglia approfondire o avvicinarsi a qualsiasi tema idrogeologico. A condire il tutto, ci saranno ovviamente delle letture plenarie condotte dai migliori specialisti dei diversi temi, secondo un programma ancora da definire nei dettagli. Sono inoltre in programma altre iniziative, i cosiddetti "side event", cioè workshop tematici condotti da organizzazioni o enti su temi specifici, che avranno luogo durante la settimana, in grado di interessare non solo i ricercatori ma anche i professionisti, secondo lo spirito della associazione.

Insomma, si preannuncia come un evento mondiale per tutti gli operatori delle acque sotterranee, e non resta che restare sintonizzati sul sito www.iah2015.org per avere tutti gli aggiornamenti fino al programma definitivo. Appuntamento per tutti a Roma dal 13 al 18 settembre!

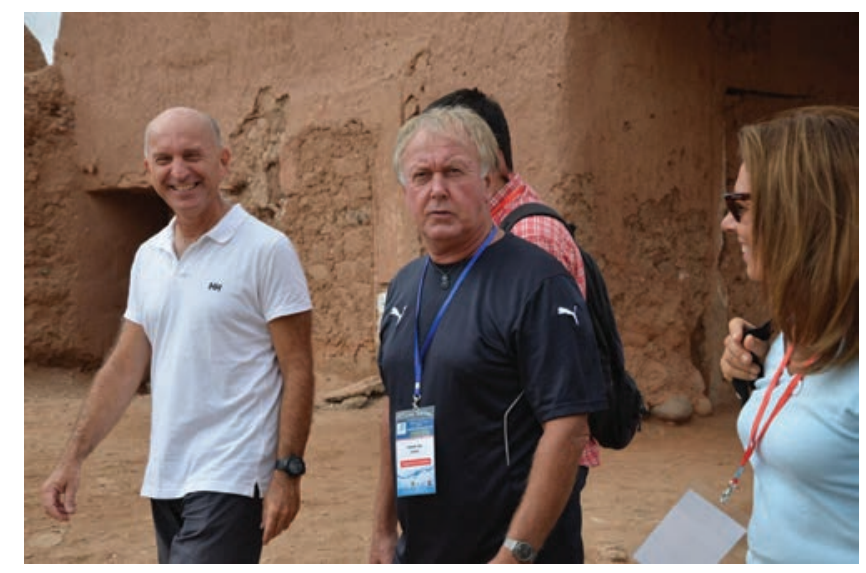

